\title{
Numerical solutions of fractional Boussinesq-Whitham- Broer-Kaup and diffusive Predator-Prey equations with conformable derivative
}

\author{
Mehmet Senol $^{1}$, Ali Kurt ${ }^{2}$, Emrah Atilgan ${ }^{3}$ and Orkun Tasbozan ${ }^{4}$ \\ ${ }^{1}$ Department of Mathematics, Faculty of Science and Art, Nevşehir Hacı Bektaş Veli University, Nevşehir, Turkey \\ ${ }^{2}$ Department of Mathematics, Faculty of Science and Literature,Pamukkale University, Denizli, Turkey \\ ${ }^{3,4}$ Department of Mathematics, Faculty of Science and Art, Mustafa Kemal University, Hatay, Turkey
}

Received: 19 May 2019, Accepted: 29 August 2019

Published online: 22 September 2019.

\begin{abstract}
In this article, the residual power series method (RPSM) is used to obtain numerical solutions of the time-fractional Boussinesq-Whitham-Broer-Kaup (BBWK) and Diffusive Predator-Prey equations using the conformable fractional derivative definition. This definition is simple, effective and reliable in the solution procedure of the fractional differential equations that have complicated solutions with classical fractional derivative definitions like Caputo and Riemann-Liouville.
\end{abstract}

Keywords: The residual power series method, time-fractional Boussinesq-Whitham-Broer-Kaup equation, time-fractional Diffusive Predator-Prey equation, conformable fractional derivative, numerical Solution.

\section{Introduction}

Fractional analysis has been the subject of numerous applications in many areas of science and engineering branches such as fractional differential equations (FDEs) [7,24], mathematical physics, biology, image and signal processing, electrical network, fluid flow, viscoelasticity and control in recent years [8,9].

There are some common methods that are used to obtain approximate or analytical solutions of nonlinear fractional ordinary and partial differential equations in the literature [28,29,30,31,32,33,34,35,36,37]. These methods include, Laplace analysis method (LAM) [15] for the constant coefficient fractional differential equations, Adomian decomposition method (ADM) [13] for the dynamics of the fractional giving up smoking model of fractional order, homotopy perturbation method (HPM) [25] for the nonlinear fractional Schrödinger equation, homotopy analysis method (HAM) [18] for the conformable fractional Nizhnik-Novikov-Veselov system, differential transformation method (DTM) [19] for the convergence of fractional power series, Elzaki projected differential transform method [22] for system of linear and nonlinear fractional partial differential equations and perturbation-iteration algoritm (PIA) [20] for ordinary fractional differential equations.

In this article, the residual power series method $[17,14,4,12,2]$ is used to obtain new approximate solutions of time-fractional Boussinesq-Whitham-Broer-Kaup equations of the form

$$
\frac{\partial^{\alpha} u}{\partial t^{\alpha}}+\frac{\partial v}{\partial x}+u \frac{\partial u}{\partial x}=0
$$




$$
\frac{\partial^{\alpha} v}{\partial t^{\alpha}}+\frac{\partial(u v)}{\partial x}+\frac{\partial^{3} u}{\partial x^{3}}=0
$$

and diffusive predator-prey equations of the form

$$
\begin{gathered}
\frac{\partial^{\alpha} u}{\partial t^{\alpha}}=\frac{\partial^{2} u}{\partial x^{2}}-\beta u+\left(\kappa+\frac{1}{\sqrt{\delta}}\right) u^{2}-u^{3}-u v \\
\frac{\partial^{\alpha} v}{\partial t^{\alpha}}=\frac{\partial^{2} v}{\partial x^{2}}+\kappa u v-\beta v-\delta v^{3}
\end{gathered}
$$

where $u=u(x, t)$ and $v=v(x, t)$. In the RPSM, the coefficients of the power series are calculated by means of the concept of residual error with the help of one or more variable algebraic equation chains, and finally, a so-called truncated series solution is obtained [17].

The major improvement of the RPSM is that it can be implemented to the problem directly without linearization, perturbation or discretization and without any transformation by selecting appropriate initial conditions [14].

After giving a few preliminary definitions and brief description of the RSPM, we presented the solution procedure of two nonlinear fractional partial differential equations that shows the reliability and efficiency of the method. Also figures and tables are presented in order to compare numerical solutions of considered equations. Finally, we discussed the obtained results in a section as conclusion.

\section{Preliminaries}

There are a few definitions of fractional derivative of order $\alpha>0$ in the literature. The most widely used are the RiemannLiouville and Caputo fractional derivatives.

Definition 1. The Riemann-Liouville fractional derivative operator $D^{\alpha} f(x)$ for $\alpha>0$ and $q-1<\alpha<q$ defined in [8,9] as:

$$
D^{\alpha} f(x)=\frac{d^{q}}{d x^{q}}\left[\frac{1}{\Gamma(q-\alpha)} \int_{\alpha}^{x} \frac{f(t)}{(x-t)^{\alpha+1-q}} d t\right]
$$

Definition 2. The Caputo fractional derivative of order $\alpha>0$ for $n \in \mathbb{N}, n-1<\alpha<n$, $D_{*}^{\alpha}$, defined in [6] as:

$$
D_{*}^{\alpha} f(x)=J^{n-\alpha} D^{n} f(x)=\frac{1}{\Gamma(n-\alpha)} \int_{\alpha}^{x}(x-t)^{n-\alpha-1}\left(\frac{d}{d t}\right)^{n} f(t) d t .
$$

Recently, a new definition of a fractional derivative called "conformable fractional derivative" has been proposed by R. Khalil et al. [16].

Definition 3. Let $f:[0, \infty) \rightarrow R$ is a function. $\alpha-$ th order "conformable fractional derivative" of function $f$ defined by

$$
T_{\alpha}(f)(t)=\lim _{\varepsilon \rightarrow 0} \frac{f\left(t+\varepsilon t^{1-\alpha}\right)-(f)(t)}{\varepsilon}
$$

for all $t>0, \alpha \in(0,1)$. The properties of this new definition are given in the following theorem [16]

Theorem 1. Let $\alpha \in(0,1]$ and $f, g$ functions are $\alpha$-differantiable at $t>0$, then 
(1) $T_{\alpha}(m f+n g)=m T_{\alpha}(f)+n T_{\alpha}(g)$ for all $m, n \in$ mathbbR

(2) $T_{\alpha}\left(t^{p}\right)=p t^{p-\alpha}$ for all $p$

(3) $T_{\alpha}(f . g)=f T_{\alpha}(g)+g T_{\alpha}(f)$

(4) $T_{\alpha}\left(\frac{f}{g}\right)=\frac{g T_{\alpha}(f)-f T_{\alpha}(g)}{g^{2}}$

(5) $T_{\alpha}(c)=0$ for all constant functions $f(t)=c$

(6) If, in addition, $f$ is differentiable, then $T_{\alpha}(f)(t)=t^{1-\alpha} \frac{d f(t)}{d t}$.

Definition 4. Let $f$ is a function with $n$ variables $x_{1}, \ldots, x_{n}$, and the conformable partial derivatives of $f$ of order $\alpha \in(0,1]$ in $x_{i}$ is defined as follows [5]

$$
\frac{d^{\alpha}}{d x_{i}^{\alpha}} f\left(x_{1}, \ldots, x_{n}\right)=\lim _{\varepsilon \rightarrow 0} \frac{f\left(x_{1}, \ldots, x_{i-1}, x_{i}+\varepsilon x_{i}^{1-\alpha}, \ldots, x_{n}\right)-f\left(x_{1}, \ldots, x_{n}\right)}{\varepsilon} .
$$

Definition 5. The conformable integral of a function $f$ for $a \geqslant 0$ is defined in [23] as

$$
I_{\alpha}^{a}(f)(s)=\int_{a}^{s} \frac{f(t)}{t^{1-\alpha}} d t
$$

\section{Description of the residual power series method}

In this section we are going to introduce some important definitions and theorems about residual power series

Theorem 2. Suppose that $f$ is an infinitely $\alpha$-differentiable function at a neigborhood of a point $t_{0}$ for some $0<\alpha \leq 1$, then $f$ has the fractional power series expansion of the form [1]:

$$
f(t)=\sum_{k=0}^{\infty} \frac{\left(T_{\alpha}^{t_{0}} f\right)^{(k)}\left(t_{0}\right)\left(t-t_{0}\right)^{k \alpha}}{\alpha^{k} k !}, t_{0}<t<t_{0}+R^{\frac{1}{\alpha}}, R>0 .
$$

Here $\left(T_{\alpha}^{t_{0}} f\right)^{(k)}\left(t_{0}\right)$ represents the application of the fractional derivative $k$-times.

Definition 6. A multiple fractional power series about $t_{0}=0$ is defined by $\sum_{n=0}^{\infty} f_{n}(x) t^{n \alpha}$ for $0 \leq m-1<\alpha<m$, where $t$ is a variable and $f_{n}(x)$ are functions called the coefficients of the series. [3, 12].

Theorem 3. Assume that $u(x, t)$ has a multiple fractional power series representation at $t_{0}=0$ of the form [3]

$$
u(x, t)=\sum_{n=0}^{\infty} f_{n}(x) t^{n \alpha}, 0 \leq m-1<\alpha<m, x \in I, 0 \leq t \leq R^{\frac{1}{\alpha}} .
$$

If $u_{t}^{(n \alpha)}(x, t), n=0,1,2, \ldots$ are continuous on $I \times\left(0, R^{\frac{1}{\alpha}}\right)$, then $f_{n}(x)=\frac{u_{t}^{(n \alpha)}(x, 0)}{\alpha^{n} n !}$

To clarify the basic concept of RPSM, let's take a nonlinear fractional differential equation of the form:

$$
T_{\alpha} u(x, t)+N[x] u(x, t)+R[x] u(x, t)=c(x, t), x \in \mathbb{R}, n-1<n \alpha \leq n, t>0
$$

expressed by initial condition

$$
f_{0}(x)=u(x, 0)=f(x)
$$


where $R[x]$ is a linear, $N[x]$ is a non-linear operator and $c(x, t)$ are continuous functions.

The RPSM method made up of stating the solution of the equation (12) subject to (13) as a fractional power series expansion around $t=0$.

$$
f_{(n-1)}(x)=T_{t}^{(n-1) \alpha} u(x, 0)=h(x)
$$

The expansion form of the solution is given by

$$
u(x, t)=f(x)+\sum_{n=0}^{\infty} f_{n}(x) \frac{t^{n \alpha}}{\alpha^{n} n !} .
$$

In the next step, the $k$.truncted series of $u(x, t)$, namely $u_{k}(x, t)$ can be written as:

$$
u_{k}(x, t)=f(x)+\sum_{n=0}^{k} f_{n}(x) \frac{t^{n \alpha}}{\alpha^{n} n !} .
$$

If the (1) RPS approximate solution $u_{1}(x, t)$ is

$$
u_{1}(x, t)=f(x)+f_{1}(x) \frac{t^{\alpha}}{\alpha^{n}}
$$

then $u_{k}(x, t)$ could be reformulated as

$$
u_{k}(x, t)=f(x)+f_{1}(x) \frac{t^{\alpha}}{\alpha^{n}}+\sum_{n=2}^{k} f_{n}(x) \frac{t^{n \alpha}}{\alpha^{n} n !}
$$

for $0<\alpha \leq 1,0 \leq t<\mathbb{R}^{\frac{1}{v}}, x \in I$ and $k=2,3,4, \ldots$ First we express the residual function as

$$
\operatorname{Res}(x, t)=T_{\alpha} u(x, t)+N[x] u(x, t)+R[x] u(x, t)-c(x, t)
$$

and the $k$. residual function as

$$
\operatorname{Res}_{k}(x, t)=T_{\alpha} u_{k}(x, t)+N[x] u_{k}(x, t)+R[x] u_{k}(x, t)-g(x, t), k=1,2,3, \ldots
$$

It is clear that $\operatorname{Res}(x, t)=0$ and $\lim _{k \rightarrow \infty} \operatorname{Res}_{k}(x, t)=\operatorname{Res}(x, t)$ for each $x \in I$ and $0 \leq t$. In fact this lead to $\frac{\partial^{(n-1) \alpha}}{\partial t^{(n-1) \alpha}} \operatorname{Res}_{k}(x, t)$ for $n=1,2,3, \ldots, k$ because in the conformable sense, the fractional derivative of a constant is zero [17,14,4]. Solving the equation $\frac{\partial^{(n-1) \alpha}}{\partial t^{(n-1) \alpha}} \operatorname{Res}_{k}(x, 0)=0$ gives us the desired $f_{n}(x)$ coefficients. Thus the $u_{n}(x, t)$ approximate solutions can be obtained respectively.

We can express the following theorem for the convergence analysis for the method [21].

Theorem 4. If there exists a fixed constant $0<K<1$ such that $\left\|u_{n+1}(x, y, t)\right\| \leq K\left\|u_{n}(x, y, t)\right\|$ for all $n \in \mathbb{N}$ and $0<t<$ $R<1$, then the sequence of approximate solution converges to an exact solution.

Proof. For all $0<t<R<1$, we have

$$
\left\|u(x, y, t)-u_{n}(x, y, t)\right\|=\left\|\sum_{i=n+1}^{\infty} u_{i}(x, y, t)\right\| \leq \sum_{i=n+1}^{\infty}\left\|u_{i}(x, y, t)\right\| \leq\|f(x, y)\| \sum_{i=n+1}^{\infty} K^{i}=\frac{K^{n+1}}{1-K}\|f(x, y)\| \stackrel{n \rightarrow \infty}{\longrightarrow} 0 .
$$




\section{Application of residual power series method}

\subsection{Solution of time-Fractional Boussinesq-Whitham-Broer-Kaup equations}

Consider the nonlinear time fractional BWBK equation

$$
\begin{gathered}
\frac{\partial^{\alpha} u}{\partial t^{\alpha}}+\frac{\partial v}{\partial x}+u \frac{\partial u}{\partial x}=0 \\
\frac{\partial^{\alpha} v}{\partial t^{\alpha}}+\frac{\partial(u v)}{\partial x}+\frac{\partial^{3} u}{\partial x^{3}}=0
\end{gathered}
$$

with the initial conditions obtained from the exact solutions as [27]

$$
\begin{gathered}
u(x, 0)=f(x)=-\frac{\omega\left(1-\tanh \left(\frac{1}{2} \sqrt{\frac{\omega^{2}}{k^{4}}} k x\right)\right)}{k} \\
v(x, 0)=g(x)=\frac{\omega^{2}\left(1-\tanh \left(\frac{1}{2} \sqrt{\frac{\omega^{2}}{k^{4}}} k x\right)\right)}{k^{2}}-\frac{\omega^{2}\left(1-\tanh \left(\frac{1}{2} \sqrt{\frac{\omega^{2}}{k^{4}}} k x\right)\right)^{2}}{2 k^{2}} .
\end{gathered}
$$

For residual power series

$$
u(x, t)=f(x)+\sum_{n=0}^{\infty} f_{n}(x) \frac{t^{n \alpha}}{\alpha^{n} n !}
$$

and $k$.truncated series of $u(x, t)$

$$
u_{k}(x, t)=f(x)+\sum_{n=0}^{k} f_{n}(x) \frac{t^{n \alpha}}{\alpha^{n} n !}, k=1,2,3, \ldots
$$

The $k_{1}$-th residual functions of time fractional BWBK equation is:

$$
\begin{gathered}
\operatorname{Resu}_{k}(x, t)=\frac{\partial^{\alpha} u_{k}}{\partial t^{\alpha}}+\frac{\partial v_{k}}{\partial x}+u_{k} \frac{\partial u_{k}}{\partial x} \\
\operatorname{Resv}_{k}(x, t)=\frac{\partial^{\alpha} v_{k}}{\partial t^{\alpha}}+\frac{\partial\left(u_{k} v_{k}\right)}{\partial x}+\frac{\partial^{3} u_{k}}{\partial x^{3}} .
\end{gathered}
$$

For determining the coefficients $f_{1}(x)$ and $g_{1}(x)$, in $u_{k}(x, t)$, we should subrogate the 1.th truncated series $u_{1}(x, t)=$ $f(x)+f_{1}(x) \frac{t^{\alpha}}{\alpha}$ and $v_{1}(x, t)=g(x)+g_{1}(x) \frac{t^{\alpha}}{\alpha}$ into the $1 s t$ truncated residual functions

$$
\begin{aligned}
\operatorname{Resu}_{1}(x, t)= & f_{1}(x)+g^{\prime}(x)+\left(f(x)+\frac{t^{\alpha} f_{1}(x)}{\alpha}\right)\left(f^{\prime}(x)+\frac{t^{\alpha} f_{1}^{\prime}(x)}{\alpha}\right)+\frac{t^{\alpha} g_{1}^{\prime}(x)}{\alpha} \\
\operatorname{Resv}_{1}(x, t)= & g_{1}(x)+f^{(3)}(x)+\frac{t^{\alpha} f_{1}^{(3)}(x)}{\alpha}+\left(g(x)+\frac{t^{\alpha} g_{1}(x)}{\alpha}\right)\left(f^{\prime}(x)+\frac{t^{\alpha} f_{1}^{\prime}(x)}{\alpha}\right) \\
& +\left(f(x)+\frac{t^{\alpha} f_{1}(x)}{\alpha}\right)\left(g^{\prime}(x)+\frac{t^{\alpha} g_{1}^{\prime}(x)}{\alpha}\right) .
\end{aligned}
$$

Now we take $t=0$ through the equations $\operatorname{Resu}_{1}(x, t)$ and $\operatorname{Resv}_{1}(x, t)$ to obtain following equations

$$
\operatorname{Resu}_{1}(x, 0)=f_{1}(x)+f(x) f^{\prime}(x)+g^{\prime}(x)
$$




$$
\operatorname{Resv}_{1}(x, 0)=g_{1}(x)+g(x) f^{\prime}(x)+f(x) g^{\prime}(x)+f^{(3)}(x) .
$$

Thus, for $\operatorname{Res}_{1}(x, 0)=0$, we get

$$
\begin{gathered}
f_{1}(x)=-f(x) f^{\prime}(x)-g^{\prime}(x) \\
g_{1}(x)=-g(x) f^{\prime}(x)-f(x) g^{\prime}(x)-f^{(3)}(x) .
\end{gathered}
$$

Therefore, we obtain the 1 st RPS approximate solutions of the time fractional BWBK equation as

$$
\begin{gathered}
u_{1}(x, t)=f(x)+\frac{t^{\alpha}\left(-f(x) f^{\prime}(x)-g^{\prime}(x)\right)}{\alpha} \\
v_{1}(x, t)=g(x)+\frac{t^{\alpha}\left(-g(x) f^{\prime}(x)-f(x) g^{\prime}(x)-f^{(3)}(x)\right)}{\alpha} .
\end{gathered}
$$

Again, to determine the second unknown coefficients $f_{2}(x)$ and $g_{2}(x)$, we subrogate the $2^{\text {nd }}$ truncated series solutions $u_{2}(x, t)=f(x)+f_{1}(x) \frac{t^{\alpha}}{\alpha}+f_{2}(x) \frac{t^{2 \alpha}}{2 \alpha^{2}}$ and $v_{2}(x, t)=g(x)+g_{1}(x) \frac{t^{\alpha}}{\alpha}+g_{2}(x) \frac{t^{2 \alpha}}{2 \alpha^{2}}$ into the $2^{\text {nd }}$ truncated residual functions to obtain following equations

$$
\begin{aligned}
\operatorname{Resu}_{2}(x, t)= & f_{1}(x)+g^{\prime}(x)+\frac{t^{\alpha} g_{1}^{\prime}(x)}{\alpha}+\frac{t^{\alpha} f_{2}(x)}{\alpha}+\frac{t^{2 \alpha} g_{2}^{\prime}(x)}{2 \alpha^{2}} \\
& +\left(f(x)+\frac{t^{\alpha} f_{1}(x)}{\alpha}+\frac{t^{2 \alpha} f_{2}(x)}{2 \alpha^{2}}\right)\left(f^{\prime}(x)+\frac{t^{\alpha} f_{1}^{\prime}(x)}{\alpha}+\frac{t^{2 \alpha} f_{2}^{\prime}(x)}{2 \alpha^{2}}\right) \\
\operatorname{Resv}_{2}(x, t)= & g_{1}(x)+f^{(3)}(x)+\frac{t^{\alpha} f_{1}^{(3)}(x)}{\alpha}+\frac{t^{2 \alpha} f_{2}^{(3)}(x)}{2 \alpha^{2}}+\frac{t^{\alpha} g_{2}(x)}{\alpha} \\
& +\left(f(x)+\frac{t^{\alpha} f_{1}(x)}{\alpha}+\frac{t^{2 \alpha} f_{2}(x)}{2 \alpha^{2}}\right)\left(g^{\prime}(x)+\frac{t^{\alpha} g_{1}^{\prime}(x)}{\alpha}+\frac{t^{2 \alpha} g_{2}^{\prime}(x)}{2 \alpha^{2}}\right) \\
& +\left(g(x)+\frac{t^{\alpha} g_{1}(x)}{\alpha}+\frac{t^{2 \alpha} g_{2}(x)}{2 \alpha^{2}}\right)\left(f^{\prime}(x)+\frac{t^{\alpha} f_{1}^{\prime}(x)}{\alpha}+\frac{t^{2 \alpha} f_{2}^{\prime}(x)}{2 \alpha^{2}}\right) .
\end{aligned}
$$

Now, applying $T_{\alpha}$ on both sides of $\operatorname{Resu}_{2}(x, t)$ and equating the equations above to zero for $t=0$ gives:

$$
\begin{gathered}
f_{2}(x)=-f_{1}(x) f^{\prime}(x)-f(x) f_{1}^{\prime}(x)-g_{1}^{\prime}(x) \\
g_{2}(x)=-g_{1}(x) f^{\prime}(x)-f_{1}(x) g^{\prime}(x)-g(x) f_{1}^{\prime}(x)-f(x) g_{1}^{\prime}(x)-f_{1}^{(3)}(x) .
\end{gathered}
$$

Therefore the 2nd RPS approximate solutions of time-fractional BWBK equation is obtained as:

$$
\begin{gathered}
u_{2}(x, t)=f(x)+\frac{t^{\alpha} f_{1}(x)}{\alpha}+\frac{t^{2 \alpha}\left(-f_{1}(x) f^{\prime}(x)-f(x) f_{1}^{\prime}(x)-g_{1}^{\prime}(x)\right)}{2 \alpha^{2}} \\
v_{2}(x, t)=g(x)+\frac{t^{\alpha} g_{1}(x)}{\alpha}+\frac{t^{2 \alpha}\left(-g_{1}(x) f^{\prime}(x)-f_{1}(x) g^{\prime}(x)-g(x) f_{1}^{\prime}(x)-f(x) g_{1}^{\prime}(x)-f_{1}^{(3)}(x)\right)}{2 \alpha^{2}} .
\end{gathered}
$$


In the same manner, we apply the same procedure for $n=3$ to obtain the following results.

$$
\begin{gathered}
f_{3}(x)=-f_{2}(x) f^{\prime}(x)-2 f_{1}(x) f_{1}^{\prime}(x)-f(x) f_{2}^{\prime}(x)-g_{2}^{\prime}(x) \\
u_{3}(x, t)=f(x)+\frac{t^{\alpha} f_{1}(x)}{\alpha}+\frac{t^{2 \alpha} f_{2}(x)}{2 \alpha^{2}}+\frac{t^{3 \alpha}\left(-f_{2}(x) f^{\prime}(x)-2 f_{1}(x) f_{1}^{\prime}(x)-f(x) f_{2}^{\prime}(x)-g_{2}^{\prime}(x)\right)}{6 \alpha^{3}} \\
g_{3}(x)=-g_{2}(x) f^{\prime}(x)-f_{2}(x) g^{\prime}(x)-2 g_{1}(x) f_{1}^{\prime}(x)-g(x) f_{2}^{\prime}(x)-2 f_{1}(x) g_{1}^{\prime}(x)-f(x) g_{2}^{\prime}(x)-f_{2}^{(3)}(x) \\
v_{3}(x, t)=g(x)+\frac{t^{\alpha} g_{1}(x)}{\alpha}+\frac{t^{2 \alpha} g_{2}(x)}{2 \alpha^{2}}+\frac{t^{3 \alpha}\left(-g_{2}(x) f^{\prime}(x)-f_{2}(x) g^{\prime}(x)\right)}{6 \alpha^{3}} \\
\frac{t^{3 \alpha}\left(-2 g_{1}(x) f_{1}^{\prime}(x)-g(x) f_{2}^{\prime}(x)-2 f_{1}(x) g_{1}^{\prime}(x)-f(x) g_{2}^{\prime}(x)-f_{2}^{(3)}(x)\right)}{6 \alpha^{3}} .
\end{gathered}
$$

In Table 1-2, the third order approximate RPSM solutions of time-fractional Boussinesq-Whitham-Broer-Kaup equations are compared numerically with exact solutions

$$
\begin{aligned}
& u(x, t)=-\frac{\omega\left(1-\tanh \left(\frac{1}{2} \sqrt{\frac{\omega^{2}}{k^{4}}} k x+\frac{t^{\alpha} \omega}{\alpha}\right)\right)}{k} \\
& v(x, t)=\frac{\omega^{2}\left(1-\tanh \left(\frac{1}{2} \sqrt{\frac{\omega^{2}}{k^{4}}} k x+\frac{t^{\alpha} \omega}{\alpha}\right)\right)}{k^{2}}-\frac{\omega^{2}\left(1-\tanh \left(\frac{1}{2} \sqrt{\frac{\omega^{2}}{k^{4}}} k x+\frac{t^{\alpha} \omega}{\alpha}\right)\right)^{2}}{2 k^{2}}
\end{aligned}
$$

for $\alpha=0.25, \alpha=0.50$ and $\alpha=0.75$ and absolute errors are presented. The results indicate that as the $x$ values increase the absolute errors increase. Besides, as the $\alpha$ values increase, the absolute errors decrease. Also the Table 1-2 show competitive solutions of the RPSM with highly approximate results. Moreover, in Figure 1-2, the surface plots of the approximate solutions are illustrated for $\alpha=0.25, \alpha=0.50, \alpha=0.75$ and $\alpha=0.95$.

Table 1: Comparison of the RPSM approximate $\left(u_{3}(x, t)\right)$ and the exact solutions with absolute errors for $\omega=0.25, k=2$, and $t=0.1$.

\begin{tabular}{|c|ccc|ccc|ccc|}
\hline & \multicolumn{3}{|c|}{$\alpha=0.25$} & & $\alpha=0.50$ & \multicolumn{3}{c|}{$\alpha=0.75$} \\
\hline$x$ & RPSM & Exact & Abs. Error & RPSM & Exact & Abs. Error & RPSM & Exact & Abs. Error \\
\hline 0.0 & -0.122804 & -0.122804 & $2.7928 \mathrm{E}-11$ & -0.124382 & -0.124382 & $4.9099 \mathrm{E}-14$ & -0.124768 & -0.124768 & $3.7470 \mathrm{E}-16$ \\
0.1 & -0.122023 & -0.122023 & $7.7575 \mathrm{E}-11$ & -0.123601 & -0.123601 & $3.5947 \mathrm{E}-13$ & -0.123987 & -0.123987 & $6.5086 \mathrm{E}-15$ \\
0.2 & -0.121242 & -0.121242 & $1.2717 \mathrm{E}-10$ & -0.12282 & -0.12282 & $6.6961 \mathrm{E}-13$ & -0.123206 & -0.123206 & $1.2628 \mathrm{E}-14$ \\
0.3 & -0.120462 & -0.120462 & $1.7668 \mathrm{E}-10$ & -0.122039 & -0.122039 & $9.7932 \mathrm{E}-13$ & -0.122425 & -0.122425 & $1.8735 \mathrm{E}-14$ \\
0.4 & -0.119682 & -0.119682 & $2.2607 \mathrm{E}-10$ & -0.121258 & -0.121258 & $1.2883 \mathrm{E}-12$ & -0.121644 & -0.121644 & $2.4855 \mathrm{E}-14$ \\
0.5 & -0.118902 & -0.118902 & $2.7532 \mathrm{E}-10$ & -0.120478 & -0.120478 & $1.5965 \mathrm{E}-12$ & -0.120864 & -0.120864 & $3.0933 \mathrm{E}-14$ \\
0.6 & -0.118123 & -0.118123 & $3.2438 \mathrm{E}-10$ & -0.119698 & -0.119698 & $1.9037 \mathrm{E}-12$ & -0.120083 & -0.120083 & $3.7012 \mathrm{E}-14$ \\
0.7 & -0.117344 & -0.117344 & $3.7322 \mathrm{E}-10$ & -0.118918 & -0.118918 & $2.2095 \mathrm{E}-12$ & -0.119304 & -0.119304 & $4.3035 \mathrm{E}-14$ \\
0.8 & -0.116566 & -0.116566 & $4.2183 \mathrm{E}-10$ & -0.118139 & -0.118139 & $2.5139 \mathrm{E}-12$ & -0.118524 & -0.118524 & $4.9058 \mathrm{E}-14$ \\
0.9 & -0.115789 & -0.115789 & $4.7015 \mathrm{E}-10$ & -0.117361 & -0.117361 & $2.8167 \mathrm{E}-12$ & -0.117745 & -0.117745 & $5.5039 \mathrm{E}-14$ \\
1.0 & -0.115012 & -0.115012 & $5.1816 \mathrm{E}-10$ & -0.116583 & -0.116583 & $3.1176 \mathrm{E}-12$ & -0.116967 & -0.116967 & $6.1006 \mathrm{E}-14$ \\
\hline
\end{tabular}


Table 2: Comparison of the RPSM approximate $\left(v_{3}(x, t)\right)$ and the exact solutions with absolute errors for $\omega=0.25, k=2$, and $t=0.1$.

\begin{tabular}{|c|ccc|ccc|ccc|}
\hline & \multicolumn{3}{|c|}{$\alpha=0.25$} & \multicolumn{3}{c|}{$\alpha=0.50$} & \multicolumn{3}{c|}{$\alpha=0.75$} \\
\hline$x$ & RPSM & Exact & Abs. Error & RPSM & Exact & Abs. Error & RPSM & Exact & Abs. Error \\
\hline 0.0 & 0.007810 & 0.007810 & $4.9661 \mathrm{E}-10$ & 0.007812 & 0.007812 & $3.1043 \mathrm{E}-12$ & 0.007812 & 0.007812 & $6.1321 \mathrm{E}-14$ \\
0.1 & 0.007808 & 0.007808 & $4.9626 \mathrm{E}-10$ & 0.007811 & 0.007811 & $3.1030 \mathrm{E}-12$ & 0.007811 & 0.007811 & $6.1298 \mathrm{E}-14$ \\
0.2 & 0.007805 & 0.007805 & $4.9558 \mathrm{E}-10$ & 0.007810 & 0.007810 & $3.0995 \mathrm{E}-12$ & 0.007810 & 0.007810 & $6.1235 \mathrm{E}-14$ \\
0.3 & 0.007802 & 0.007802 & $4.9458 \mathrm{E}-10$ & 0.007808 & 0.007808 & $3.0941 \mathrm{E}-12$ & 0.007809 & 0.007809 & $6.1131 \mathrm{E}-14$ \\
0.4 & 0.007798 & 0.007798 & $4.9324 \mathrm{E}-10$ & 0.007805 & 0.007805 & $3.0866 \mathrm{E}-12$ & 0.007806 & 0.007806 & $6.0986 \mathrm{E}-14$ \\
0.5 & 0.007793 & 0.007793 & $4.9158 \mathrm{E}-10$ & 0.007802 & 0.007802 & $3.0770 \mathrm{E}-12$ & 0.007803 & 0.007803 & $6.0802 \mathrm{E}-14$ \\
0.6 & 0.007788 & 0.007788 & $4.8959 \mathrm{E}-10$ & 0.007798 & 0.007798 & $3.0654 \mathrm{E}-12$ & 0.007800 & 0.007800 & $6.0575 \mathrm{E}-14$ \\
0.7 & 0.007783 & 0.007783 & $4.8729 \mathrm{E}-10$ & 0.007794 & 0.007794 & $3.0518 \mathrm{E}-12$ & 0.007796 & 0.007796 & $6.0312 \mathrm{E}-14$ \\
0.8 & 0.007776 & 0.007776 & $4.8466 \mathrm{E}-10$ & 0.007788 & 0.007788 & $3.0362 \mathrm{E}-12$ & 0.007791 & 0.007791 & $6.0006 \mathrm{E}-14$ \\
0.9 & 0.007770 & 0.007770 & $4.8172 \mathrm{E}-10$ & 0.007783 & 0.007783 & $3.0186 \mathrm{E}-12$ & 0.007786 & 0.007786 & $5.9663 \mathrm{E}-14$ \\
1.0 & 0.007762 & 0.007762 & $4.7846 \mathrm{E}-10$ & 0.007777 & 0.007777 & $2.9990 \mathrm{E}-12$ & 0.007780 & 0.007780 & $5.9281 \mathrm{E}-14$ \\
\hline
\end{tabular}

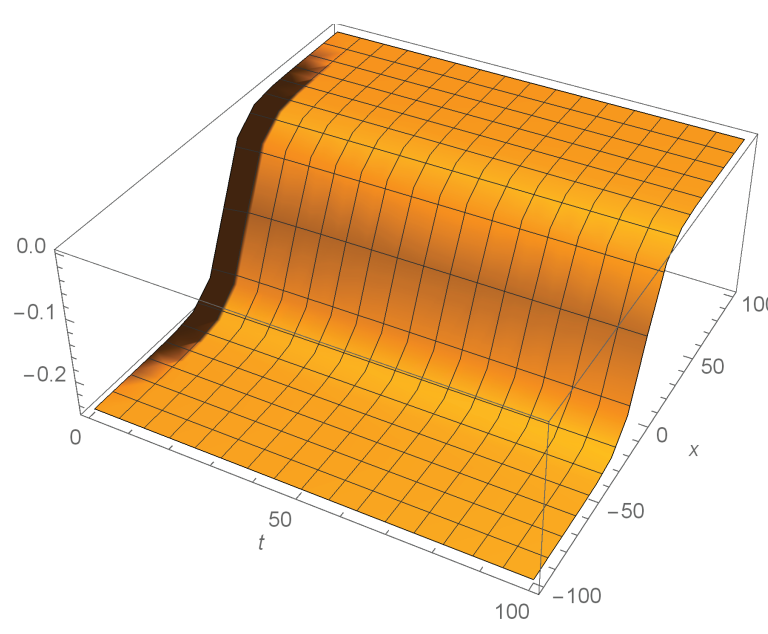

(a)

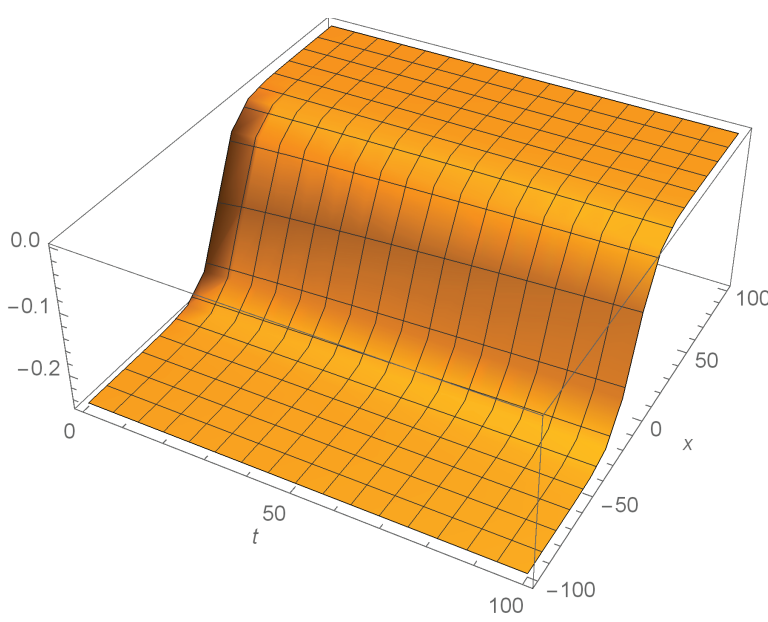

(c)

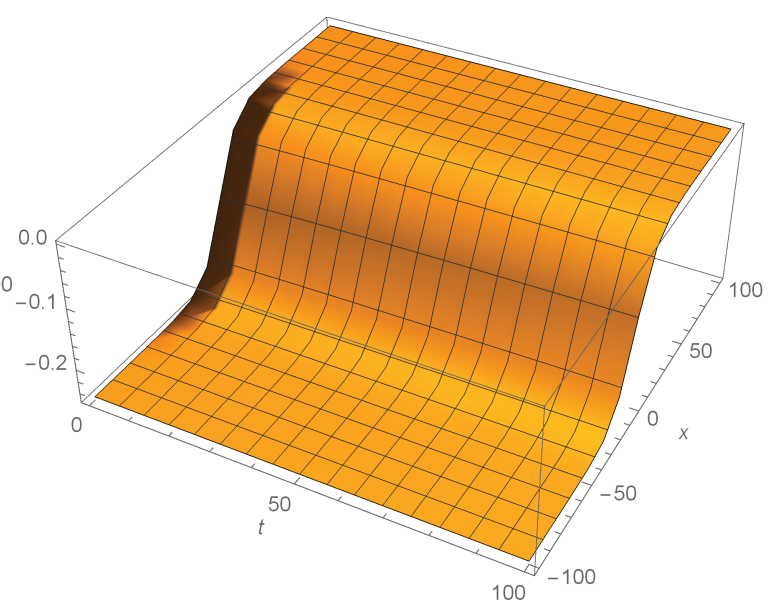

(b)

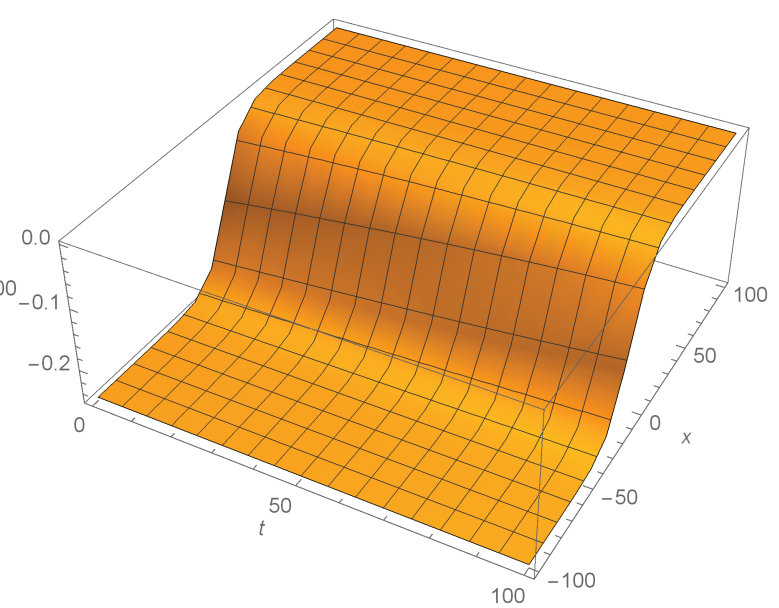

(d)

Fig. 1: The surface plots of $u_{3}(x, t)$ for $\omega=0.25, k=2, t=0.1$ and for (a) $\alpha=0.25$, (b) $\alpha=0.50$, (c) $\alpha=0.75$, (d) $\alpha=0.95$. 


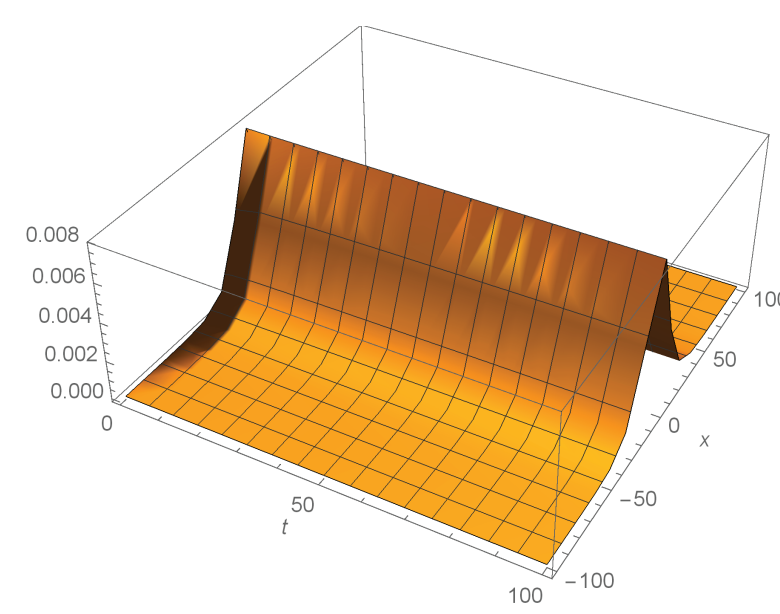

(a)

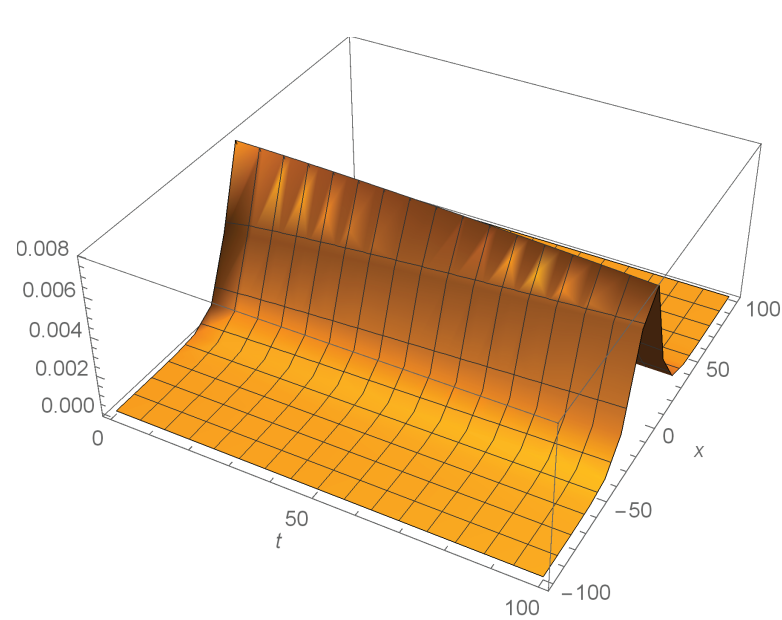

(c)

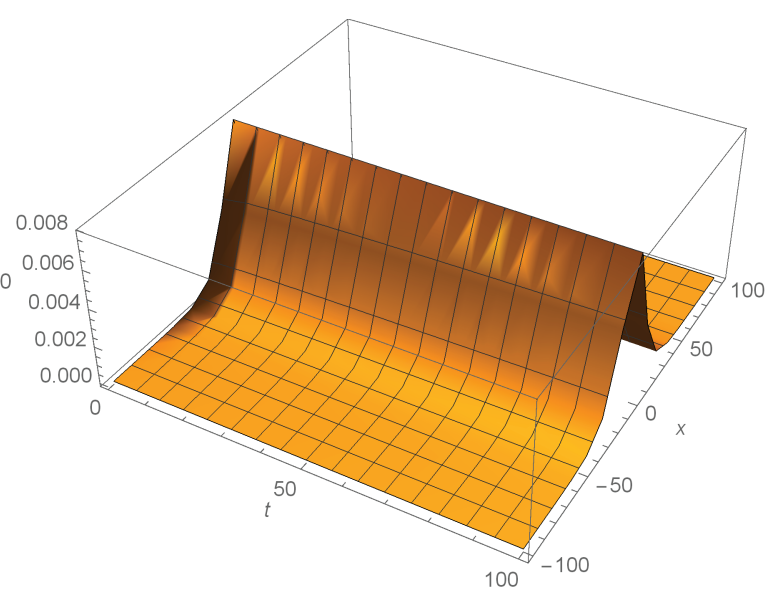

(b)

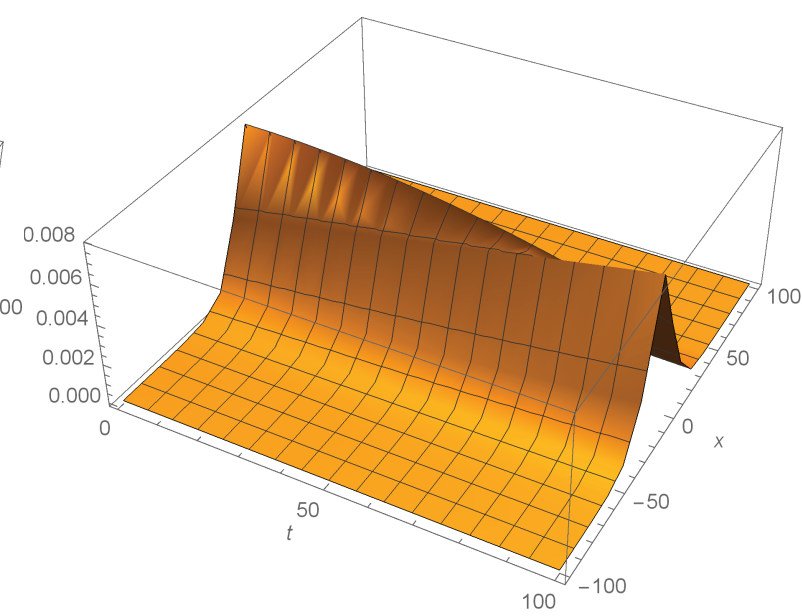

(d)

Fig. 2: The surface plots of $v_{3}(x, t)$ for $\omega=0.25, k=2, t=0.1$ and for (a) $\alpha=0.25$, (b) $\alpha=0.50$, (c) $\alpha=0.75$, (d) $\alpha=0.95$.

\subsection{Solution of time-Fractional Predator-Prey Equations}

Now consider the time-fractional diffusive predator-prey equtions of the form

$$
\begin{gathered}
\frac{\partial^{\alpha} u}{\partial t^{\alpha}}=\frac{\partial^{2} u}{\partial x^{2}}-\beta u+\left(\kappa+\frac{1}{\sqrt{\delta}}\right) u^{2}-u^{3}-u v \\
\frac{\partial^{\alpha} v}{\partial t^{\alpha}}=\frac{\partial^{2} v}{\partial x^{2}}+\kappa u v-\beta v-\delta v^{3}
\end{gathered}
$$

with the initial conditions

$$
\begin{gathered}
u(x, 0)=f(x)=\frac{v}{\sqrt{2}}+\sqrt{2} \tanh x \\
v(x, 0)=g(x)=\frac{1}{\sqrt{\delta}}\left(\frac{v}{\sqrt{2}}+\sqrt{2} \tanh x\right) .
\end{gathered}
$$


Now repeating the above residual power series proedure we obtained the following results.

$$
\begin{aligned}
& f_{1}(x)=-\beta f(x)+\left(\frac{1}{\sqrt{\delta}}+\kappa\right) f^{2}(x)-f^{3}(x)-f(x) g(x)+f^{\prime \prime}(x) \\
& u_{1}(x, t)=f(x)+\frac{t^{\alpha}}{\alpha}\left(-\beta f(x)+\left(\frac{1}{\sqrt{\delta}}+\kappa\right) f^{2}(x)-f^{3}(x)-f(x) g(x)+f^{\prime \prime}(x)\right) \\
& g_{1}(x)=-\beta g(x)+\kappa f(x) g(x)-\delta g^{3}(x)+g^{\prime \prime}(x) \\
& v_{1}(x, t)=g(x)+\frac{t^{\alpha}}{\alpha}\left(-\beta g(x)+\kappa f(x) g(x)-\delta g^{3}(x)+g^{\prime \prime}(x)\right) \\
& f_{2}(x)=-\beta f_{1}(x)+2\left(\frac{1}{\sqrt{\delta}}+\kappa\right) f(x) f_{1}(x)-3 f^{2}(x) f_{1}(x)-f_{1}(x) g(x)-f(x) g_{1}(x)+f^{\prime \prime}(x) \\
& u_{2}(x, t)=f(x)+\frac{t^{\alpha} f_{1}(x)}{\alpha}+\frac{t^{2 \alpha}}{2 \alpha^{2}}\left(-\beta f_{1}(x)+2\left(\frac{1}{\sqrt{\delta}}+\kappa\right) f(x) f_{1}(x)\right) \\
& \frac{t^{2 \alpha}}{2 \alpha^{2}}\left(-3 f^{2}(x) f_{1}(x)-f_{1}(x) g(x)-f(x) g_{1}(x)+f^{\prime \prime}(x)\right) \\
& g_{2}(x)=\kappa f_{1}(x) g(x)-\beta g_{1}(x)+\kappa f(x) g_{1}(x)-3 \delta g^{2}(x) g_{1}(x)+g_{1}^{\prime \prime}(x) \\
& v_{2}(x, t)=g(x)+\frac{t^{\alpha} g_{1}(x)}{\alpha}+\frac{t^{2 \alpha}}{2 \alpha^{2}}\left(\kappa f_{1}(x) g(x)-\beta g_{1}(x)+\kappa f(x) g_{1}(x)-3 \delta g^{2}(x) g_{1}(x)+g_{1}^{\prime \prime}(x)\right) \\
& f_{3}(x)=\frac{1}{\sqrt{\delta}}\left(2 f_{1}^{2}(x)+2 \sqrt{\delta} \kappa f_{1}^{2}(x)-6 \sqrt{\delta} f(x) f_{1}^{2}(x)-\beta \sqrt{\delta} f_{2}(x)+2 f(x) f_{2}(x)+2 \sqrt{\delta} \kappa f(x) f_{2}(x)\right) \\
& -\frac{1}{\sqrt{\delta}}\left(3 \sqrt{\delta} f^{2}(x) f_{2}(x)+\sqrt{\delta} g(x) f_{2}(x)-2 \sqrt{\delta} f_{1}(x) g_{1}(x)-\sqrt{\delta} f(x) g_{2}(x)+\sqrt{\delta} f_{2}^{\prime \prime}(x)\right) \\
& u_{3}(x, t)=f(x)+\frac{t^{\alpha} f_{1}(x)}{\alpha}+\frac{t^{2 \alpha} f_{2}(x)}{2 \alpha^{2}}+\frac{1}{\sqrt{\delta}} \frac{t^{3 \alpha}}{6 \alpha^{3}}\left(2 f_{1}^{2}(x)+2 \sqrt{\delta} \kappa f_{1}^{2}(x)-6 \sqrt{\delta} f(x) f_{1}^{2}(x)\right) \\
& -\frac{1}{\sqrt{\delta}} \frac{t^{3 \alpha}}{6 \alpha^{3}}\left(-\beta \sqrt{\delta} f_{2}(x)+2 f(x) f_{2}(x)+2 \sqrt{\delta} \kappa f(x) f_{2}(x) 3 \sqrt{\delta} f^{2}(x) f_{2}(x)\right) \\
& +\frac{1}{\sqrt{\delta}} \frac{t^{3 \alpha}}{6 \alpha^{3}}\left(\sqrt{\delta} g(x) f_{2}(x)-2 \sqrt{\delta} f_{1}(x) g_{1}(x)-\sqrt{\delta} f(x) g_{2}(x)+\sqrt{\delta} f_{2}^{\prime \prime}(x)\right) \\
& g_{3}(x)=\kappa g(x) f_{2}(x)+2 \kappa f_{1}(x) g_{1}(x)-6 \delta g(x) g_{1}^{2}(x)-\beta g_{2}(x)+\kappa f(x) g_{2}(x)-3 \delta g^{2}(x) g_{2}(x)+g_{2}^{\prime \prime}(x) \\
& v_{3}(x, t)=g(x)+\frac{t^{\alpha} g_{1}(x)}{\alpha}+\frac{t^{2 \alpha} g_{2}(x)}{2 \alpha^{2}}+\frac{t^{3 \alpha}}{2 \alpha^{2}}\left(\kappa g(x) f_{2}(x)+2 \kappa f_{1}(x) g_{1}(x)\right) \\
& +\frac{t^{3 \alpha}}{2 \alpha^{2}}\left(-6 \delta g(x) g_{1}^{2}(x)-\beta g_{2}(x)+\kappa f(x) g_{2}(x)-3 \delta g^{2}(x) g_{2}(x)+g_{2}^{\prime \prime}(x)\right) \text {. }
\end{aligned}
$$


In Table 3-4, the third order approximate RPSM solutions of time-fractional Predator-Prey equations are compared numerically with the exact solutions [26]

$$
\begin{gathered}
u(x, t)=\frac{v}{\sqrt{2}}+\sqrt{2} \tanh \left(x+\frac{t^{\alpha} v}{\alpha}\right) \\
v(x, t)=\frac{1}{\sqrt{\delta}}\left(\frac{v}{\sqrt{2}}+\sqrt{2} \tanh \left(x+\frac{t^{\alpha} v}{\alpha}\right)\right)
\end{gathered}
$$

for $\alpha=0.75, \alpha=0.85$ and $\alpha=0.95$ and absolute errors are presented. The results show that as the absolute value of the $x$ points increase the absolute errors decrease. Besides, as the $\alpha$ values increase, the absolute errors decrease. Also in Figure 3 and 4 , the surface plots of the approximate solutions are illustrated for $\alpha=0.65, \alpha=0.75, \alpha=0.85$ and $\alpha=0.95$.

Table 3: Comparison of the RPSM approximate $\left(u_{3}(x, t)\right)$ and the exact solutions with absolute errors for $\delta=0.25, \kappa=2$,

\begin{tabular}{|c|c|c|c|c|c|c|c|c|c|}
\hline & \multicolumn{3}{|c|}{$\alpha=0.75$} & \multicolumn{3}{|c|}{$\alpha=0.85$} & \multicolumn{3}{|c|}{$\alpha=0.95$} \\
\hline$x$ & RPSM & Exact & Abs. Error & RPSM & Exact & Abs. Error & $\overline{R P S M}$ & Exact & Abs. Error \\
\hline-10 & -0.414213 & -0.414213 & $5.6537 \mathrm{E}-11$ & -0.414214 & -0.414213 & $1.3064 \mathrm{E}-11$ & -0.414213 & -0.414213 & $3.2387 \mathrm{E}-12$ \\
\hline-8 & -0.414212 & -0.414212 & $3.0868 \mathrm{E}-9$ & -0.414213 & -0.414213 & $7.1328 \mathrm{E}-10$ & -0.414213 & -0.414213 & $1.7684 \mathrm{E}-10$ \\
\hline-6 & -0.414179 & -0.414179 & $1.6851 \mathrm{E}-7$ & -0.414186 & -0.414185 & $3.8940 \mathrm{E}-8$ & -0.414189 & -0.414189 & $9.6541 \mathrm{E}-9$ \\
\hline-4 & -0.412368 & -0.412359 & $9.1442 \mathrm{E}-6$ & -0.412698 & 0.412696 & $2.1136 \mathrm{E}-6$ & -0.412889 & -0.412889 & $5.2412 \mathrm{E}-7$ \\
\hline-2 & -0.316762 & -0.316414 & $3.4829 \mathrm{E}-4$ & -0.333766 & -0.333684 & $8.202 \mathrm{E}-5$ & -0.343687 & -0.343666 & $2.0573 \mathrm{E}-5$ \\
\hline 0 & 1.456435 & 1.457199 & $7.6453 \mathrm{E}-4$ & 1.326242 & 1.326374 & $1.3223 \mathrm{E}-4$ & 1.234017 & 1.234041 & $2.4240 \mathrm{E}-5$ \\
\hline 2 & 2.388259 & 2.387967 & $2.9187 \mathrm{E}-4$ & 2.382275 & 2.382203 & $7.2407 \mathrm{E}-5$ & 2.377619 & 2.377601 & $1.8822 \mathrm{E}-5$ \\
\hline 4 & 2.413735 & 2.413728 & 6.9999E-6 & 2.413622 & 2.413620 & $1.7529 \mathrm{E}-6$ & 2.413534 & 2.413534 & 4.5887E-7 \\
\hline 6 & 2.414204 & 2.414204 & $1.2881 \mathrm{E}-7$ & 2.4142027 & 2.414202 & $3.2261 \mathrm{E}-8$ & 2.414201 & 2.414201 & $8.4462 \mathrm{E}-9$ \\
\hline 8 & 2.414213 & 2.414213 & $2.3594 \mathrm{E}-9$ & 2.414213 & 2.414213 & $5.9094 \mathrm{E}-10$ & 2.414213 & 2.414213 & $1.5471 \mathrm{E}-10$ \\
\hline 10 & 2.414213 & 2.414213 & $4.3215 \mathrm{E}-11$ & 2.414213 & 2.414213 & $1.0823 \mathrm{E}-11$ & 2.414213 & 2.414213 & $2.8337 \mathrm{E}-12$ \\
\hline
\end{tabular}
$\beta=-1, v=\sqrt{2}$ and $t=0.1$.

Table 4: Comparison of the RPSM approximate $\left(v_{3}(x, t)\right)$ and the exact solutions with absolute errors for $\delta=0.25, \kappa=2$,

\begin{tabular}{|c|c|c|c|c|c|c|c|c|c|}
\hline & \multicolumn{3}{|c|}{$\alpha=0.75$} & \multicolumn{3}{|c|}{$\alpha=0.85$} & \multicolumn{3}{|c|}{$\alpha=0.95$} \\
\hline$x$ & $R P S M$ & Exact & Abs. Error & RPSM & Exact & Abs. Error & $R P S M$ & Exact & Abs. Error \\
\hline-10 & -0.828427 & -0.828427 & $1.1307 \mathrm{E}-10$ & -0.828427 & -0.828427 & $2.6128 \mathrm{E}-11$ & -0.828427 & -0.828427 & $6.4776 \mathrm{E}-12$ \\
\hline-8 & -0.828425 & -0.828425 & $6.1736 \mathrm{E}-9$ & -0.828426 & -0.828426 & $1.4265 \mathrm{E}-9$ & -0.828426 & -0.828426 & $3.5368 \mathrm{E}-10$ \\
\hline-6 & -0.828359 & -0.828359 & $3.3703 \mathrm{E}-7$ & -0.828371 & -0.828371 & $7.7880 \mathrm{E}-8$ & -0.828378 & -0.828378 & $1.9308 \mathrm{E}-8$ \\
\hline-4 & -0.824737 & -0.824718 & $1.8288 \mathrm{E}-5$ & -0.825396 & -0.825392 & 4.2273E-6 & -0.825779 & -0.825778 & $1.0482 \mathrm{E}-6$ \\
\hline-2 & -0.633525 & -0.632828 & $6.9659 \mathrm{E}-4$ & -0.667532 & -0.667368 & $16405 \mathrm{E}-4$ & -0.687374 & -0.687333 & $4.1147 \mathrm{E}-5$ \\
\hline 0 & 2.912870 & 2.914399 & $1.5290 \mathrm{E}-3$ & 2.652485 & 2.652749 & $26446 \mathrm{E}-4$ & 2.468035 & 2.468083 & $4.8480 \mathrm{E}-5$ \\
\hline 2 & 4.776518 & 4.775934 & $5.8375 \mathrm{E}-4$ & 4.764550 & 4.764406 & $14481 \mathrm{E}-4$ & 4.755239 & 4.755202 & $3.7644 \mathrm{E}-5$ \\
\hline 4 & 4.827470 & 4.827456 & $1.3999 \mathrm{E}-5$ & 4.827244 & 4.827241 & $3.5058 \mathrm{E}-6$ & 4.827069 & 4.827068 & $9.1774 \mathrm{E}-7$ \\
\hline 6 & 4.828409 & 4.828409 & $2.5762 \mathrm{E}-7$ & 4.828405 & 4.828405 & $6.4522 \mathrm{E}-8$ & 4.828402 & 4.828402 & $1.6892 \mathrm{E}-8$ \\
\hline 8 & 4.828426 & 4.828426 & $4.7189 \mathrm{E}-9$ & 4.828426 & 4.828426 & $1.1818 \mathrm{E}-9$ & 4.828426 & 4.828426 & $3.0942 \mathrm{E}-10$ \\
\hline 10 & 4.828427 & 4.828427 & $8.6430 \mathrm{E}-11$ & 4.828427 & 4.828427 & $2.1646 \mathrm{E}-11$ & 4.828427 & 4.828427 & $5.6665 \mathrm{E}-12$ \\
\hline
\end{tabular}
$\beta=-1, v=\sqrt{2}$ and $t=0.1$.

\section{Conclusion}

In this paper, approximate solutions of the nonlinear time-fractional Boussinesq-Whitham-Broer-Kaup and Diffusive Predator-Prey equations are obtained by the residual power series method (RPSM) . With conformable fractional derivative definition, we can easily transform fractional differential equations to the known classical differential equations. By the presented method and the conformable fractional derivative definition, it is shown that there is no need 


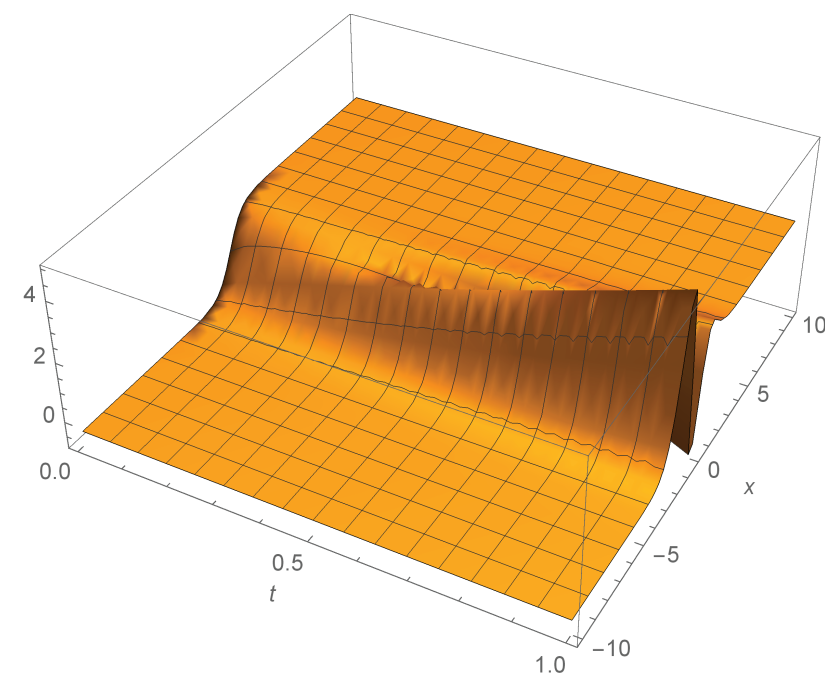

(a)

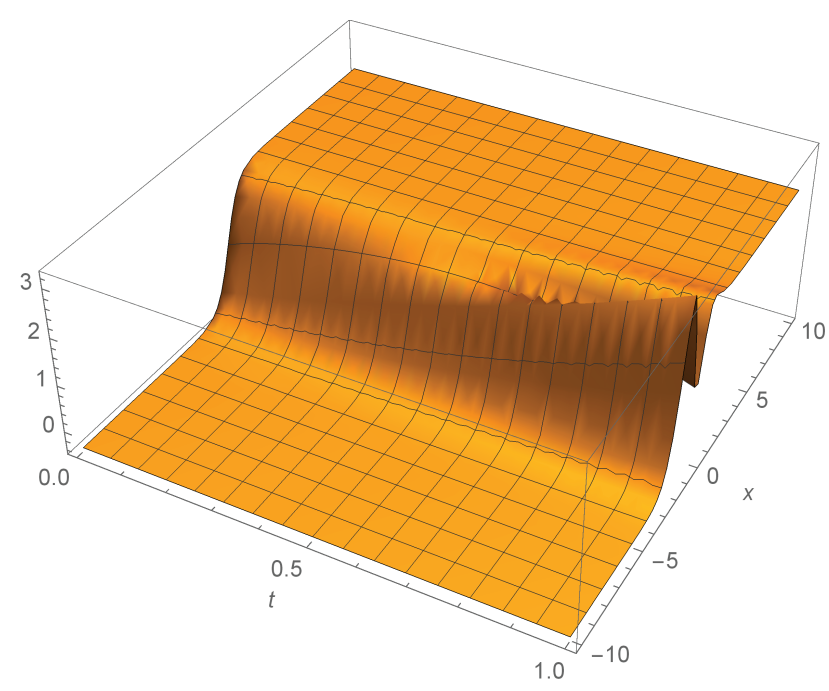

(c)

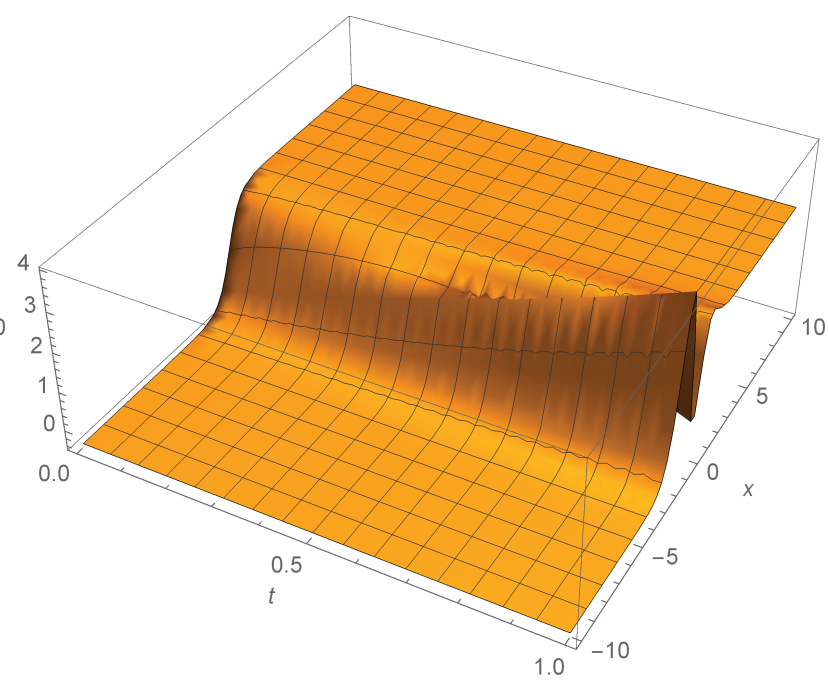

(b)

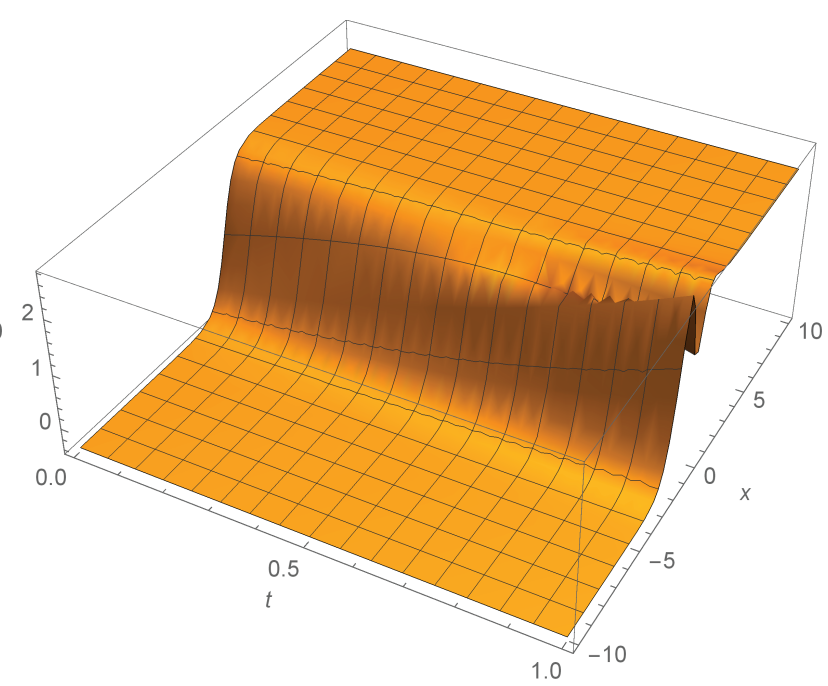

(d)

Fig. 3: The surface plots of $u_{3}(x, t)$ for $\delta=0.25, \kappa=2, \beta=-1, v=\sqrt{2}, t=0.1$ and for (a) $\alpha=0.65$, (b) $\alpha=0.75$, (c) $\alpha=0.85$, (d) $\alpha=0.95$.

to another complex method or definition. Approximate and exact solutions of time-fractional nonlinear differential equations are compered to show the reliability of the method. Absolute errors were given with approximate and exact solutions with the help of figures and tables. Also our work reported here is a first step towards understanding structural and physical behaviour of mathematical models in biology. We hope that our work will be very useful in better understanding the events occurring at mathematical biology.

\section{Competing interests}

The authors declare that they have no competing interests. 


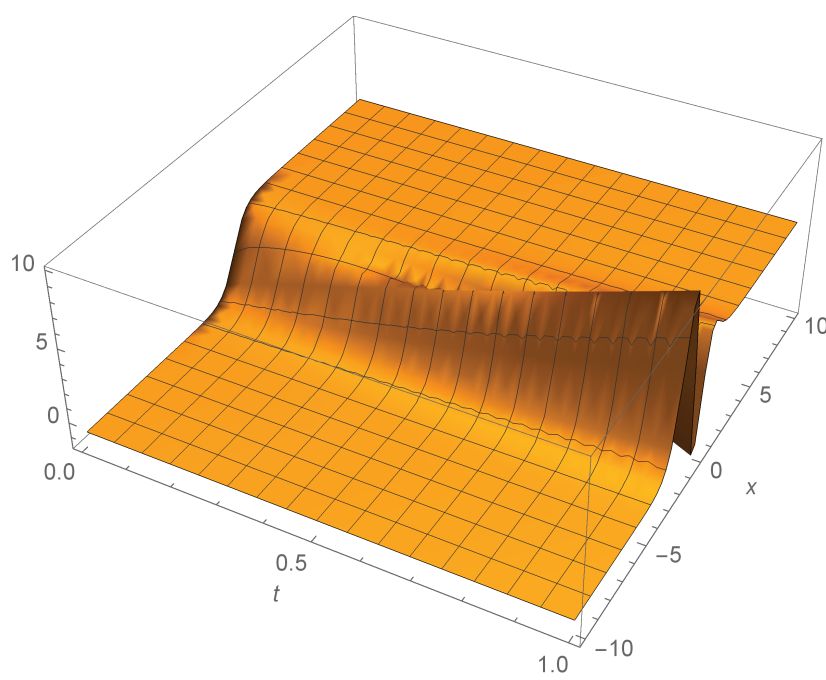

(a)

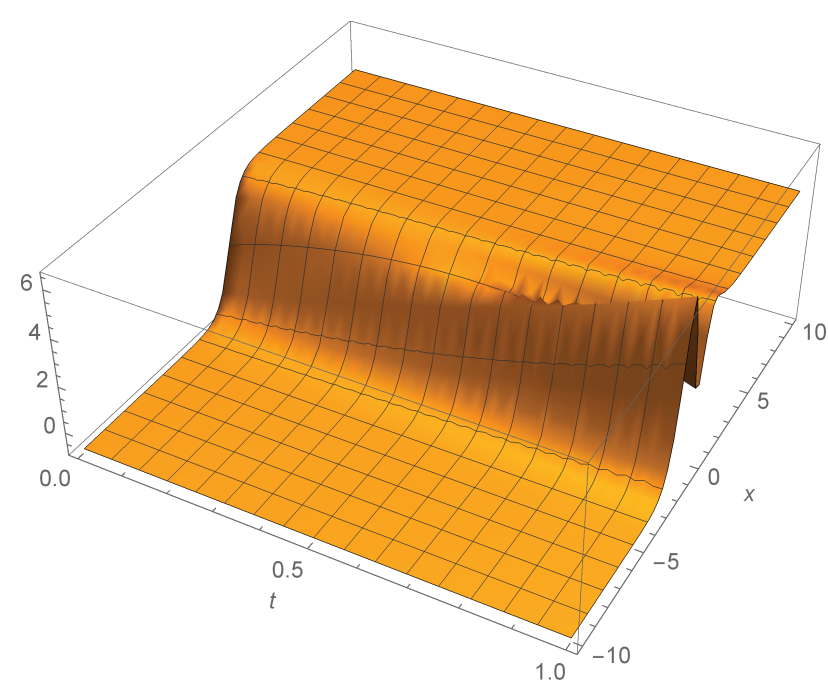

(c)

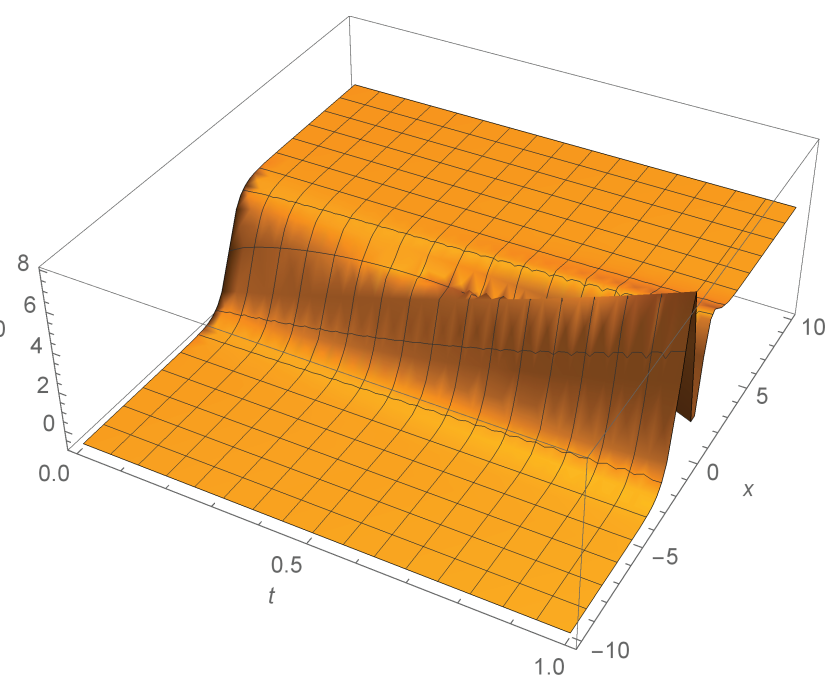

(b)

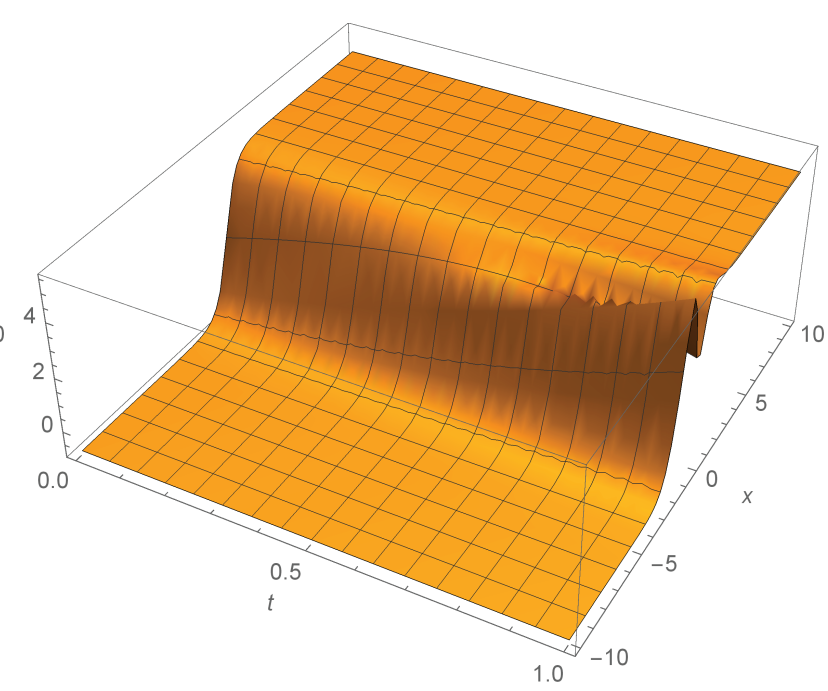

(d)

Fig. 4: The surface plots of $v_{3}(x, t)$ for $\delta=0.25, \kappa=2, \beta=-1, v=\sqrt{2}, t=0.1$ and for (a) $\alpha=0.65$, (b) $\alpha=0.75$, (c) $\alpha=0.85$, (d) $\alpha=0.95$.

\section{Authors' contributions}

All authors have contributed to all parts of the article. All authors read and approved the final manuscript.

\section{References}

[1] Abdeljawad, T, "On conformable fractional calculus." Journal of computational and Applied Mathematics 279 (2015): 57-66.

[2] Ahmad, R. S. "An analytical solution of the fractional Navier-Stokes equation by residual power series method.", Zarqa University, Doctoral dissertation, 10-90, 2015.

[3] Alabsi T.Y., "Solution of Conformable Fractional Navier-Stokes Equation", M.S. thesis, Zarqa University, 2017. 
[4] Alquran, M. "Analytical solutions of fractional foam drainage equation by residual power series method", Mathematical sciences, $8(4), 153,2015$.

[5] Atangana, Abdon, Dumitru Baleanu, and Ahmed Alsaedi. "New properties of conformable derivative." Open Mathematics 13.1 (2015).

[6] Caputo, M., "Linear models of dissipation whose Q is almost frequency independent-II", Geophys. J. Int., 13.5, 529-539, 1967.

[7] Çelik, C., and Melda Duman. "Finite element method for a symmetric tempered fractional diffusion equation." Applied Numerical Mathematics 120 (2017): 270-286.

[8] S.G. Samko, A.A. Kilbas, and O.I. Marichev, Fractional Integrals and Derivatives: Theory and Applications, Gordon and Breach, Yverdon (1993).

[9] I. Podlubny, Fractional Differential Equations, Academic Press, San Diego (1999).

[10] Das, S., "Functional fractional calculus", Springer, 2011.

[11] Diethelm, K., "The analysis of fractional differential equations", Springer,2010

[12] El-Ajou, A., Arqub, O. A., Zhour, Z. A., \& Momani, S. "New results on fractional power series: theories and applications", Entropy, 15(12), 5305-5323, 2013.

[13] Haq, F., Shah, K., ur Rahman, G., and Shahzad, M. "Numerical solution of fractional order smoking model via laplace Adomian decomposition method". Alexandria Engineering Journal. (2017).

[14] Jaradat, H. M., Al-Shara, S., Khan, Q. J., Alquran, M., \& Al-Khaled, K. "Analytical solution of time-fractional Drinfeld-SokolovWilson system using residual power series method”, IAENG International Journal of Applied Mathematics, 46(1), 64-70, 2016.

[15] Kexue, L., \& Jigen, P. (2011). Laplace transform and fractional differential equations. Applied Mathematics Letters, 24(12), 20192023.

[16] Khalil, Roshdi, et al. "A new definition of fractional derivative.”Journal of Computational and Applied Mathematics 264 (2014): 65-70.

[17] Kumar, A., Kumar, S., \& Singh, M., "Residual power series method for fractional Sharma-Tasso-Olever equation", Commun. Numer. Anal, 10, 2016.

[18] Kurt, A., O. Tasbozan, and D. Baleanu. "New solutions for conformable fractional Nizhnik-Novikov-Veselov system via $G$ / $/ G$ expansion method and homotopy analysis methods." Optical and Quantum Electronics 49.10 (2017): 333.

[19] Odibat, Z. M., Kumar, S., Shawagfeh, N., Alsaedi, A., and Hayat, T., ”A study on the convergence conditions of generalized differential transform method". Mathematical Methods in the Applied Sciences, 40(1)(2017), 40-48.

[20] Şenol, M., and Dolapci, I.T. “On the Perturbation-Iteration Algorithm for fractional differential equations”, Journal of King Saud University-Science, 28(1), 69-74, 2016.

[21] Şenol, M., Alquran, M., Kasmaei, H.D., "On the comparison of perturbation-iteration algorithm and residual power series method to solve fractional Zakharov-Kuznetsov equation", Results in Physics, 9(2018), 321-327.

[22] Suleman, M., Lu, D., He, J.H., Farooq, U., Noeiaghdam, S., and Chandio, F.A. "Elzaki Projected Differential Transform method for Fractional order System of Linear and Nonlinear Fractional Partial Differential Equation", Fractals, https://doi.org/10.1142/S0218348X1850041X.

[23] Tasbozan, O., Çenesiz, Y., and Kurt, A., ”New solutions for conformable fractional Boussinesq and combined KdV-mKdV equations using Jacobi elliptic function expansion method". The European Physical Journal Plus, 131(7) (2016), 244.

[24] Yakar, A., and Kutlay, H. "Monotone Iterative Technique via Initial Time Different Coupled Lower and Upper Solutions for Fractional Differential Equations." Filomat 31.4 (2017): 1031-1039.

[25] Yildirim, A. (2009). An algorithm for solving the fractional nonlinear Schrödinger equation by means of the homotopy perturbation method. International Journal of Nonlinear Sciences and Numerical Simulation, 10(4), 445-450.

[26] Kurt A., Eslami M., Rezazadeh H., Tasbozan O., Ozkan O., (2019) The New Exact and Approximate Solution for the Nonlinear Fractional Diffusive Predator-Prey system Arising in Mathematical Biology, Journal of New Theory, 28, 33-43.

[27] Atilgan E., Senol M., Kurt A., Tasbozan O. (2019) New Wave Solutions of Time Fractional Coupled Boussinesq-Whitham-BroerKaup Equation as a Model of Water Waves, Applied Mathematics and Nonlinear Sciences (In Press).

[28] Rizvi, S. T. R., Ali, K., Akram, U., Younis, M. (2018). Analytical study of solitons for Lakshmanan Porsezian Daniel model with parabolic law nonlinearity. Optik, 168, 27-33.

[29] Rizvi, S. T. R., Ali, K., Hanif, H. (2019). Optical solitons in dual core fibers under various nonlinearities. Modern Physics Letters B, 1950189. 
[30] Nawaz, B., Ali, K., Abbas, S. O., Rizvi, S. T. R., Zhou, Q. (2019). Optical solitons for non-Kerr law nonlinear Schrödinger equation with third and fourth order dispersions. Chinese Journal of Physics, 60, 133-140.

[31] Tariq, K. U., Younis, M., Rezazadeh, H., Rizvi, S. T. R., Osman, M. S. (2018). Optical solitons with quadratic cubic nonlinearity and fractional temporal evolution. Modern Physics Letters B, 32(26), 1850317.

[32] Rezazadeh, H., Osman, M. S., Eslami, M., Mirzazadeh, M., Zhou, Q., Badri, S. A., Korkmaz, A. (2019). Hyperbolic rational solutions to a variety of conformable fractional Boussinesq-Like equations. Nonlinear Engineering, 8(1), 224-230.

[33] Osman, M. S. (2019). New analytical study of water waves described by coupled fractional variant Boussinesq equation in fluid dynamics. Pramana, 93(2), 26.

[34] Osman M.S., Rezazadeh H., Eslami M. (2019). Traveling wave solutions for (3+1) dimensional conformable fractional Zakharov Kuznetsov equation with power law nonlinearity. Nonlinear Engineering, 8(1), 559-567.

[35] Rezazadeh, H., Osman, M. S., Eslami, M., Ekici, M., Sonmezoglu, A., Asma, M., Othman W.A.M., Wong B.R., Mirzazadeh M., Zhou Q., Biswas, A. (2018). Mitigating Internet bottleneck with fractional temporal evolution of optical solitons having quadratic cubic nonlinearity. Optik, 164, 84-92.

[36] Osman, M. S., Ghanbari, B. (2018). New optical solitary wave solutions of Fokas-Lenells equation in presence of perturbation terms by a novel approach. Optik, 175, 328-333.

[37] Osman, M. S. (2017). Multiwave solutions of time-fractional (2+ 1)-dimensional Nizhnik-Novikov-Veselov equations. Pramana, $88(4), 67$. 